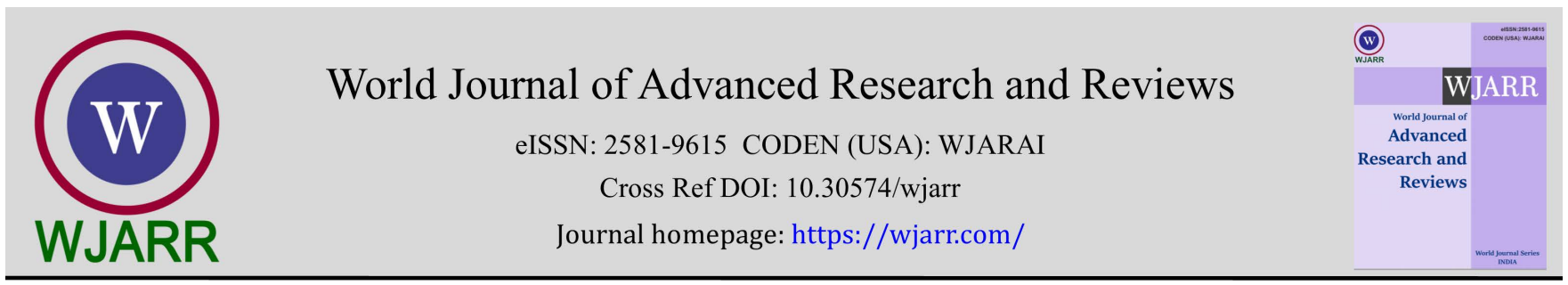

(RESEARCH ARTICLE)

\title{
Prognosis of COVID-19 patients with type 2 diabetes
}

\author{
Brunda M 1, Spandana Peddareddy 1, Arushi Mohan 1, *, Mahesh DM 2, Samriddha Thapa 3, \\ Harshadayani Jagadish Kumar ${ }^{1}$ and GR Lingeshwaran ${ }^{1}$
}

${ }^{1}$ Department of Internal Medicine, ASTER CMI Hospital, Hebbal, Bangalore, India.

${ }^{2}$ Department of Endocrinology, ASTER CMI Hospital, Hebbal, Bangalore, India.

${ }^{3}$ Patan Academy of Health Sciences - School of Medicine, Nepal.

World Journal of Advanced Research and Reviews, 2021, 12(01), 126-139

Publication history: Received on 27 August 2021; revised on 03 October 2021; accepted on 05 October 2021

Article DOI: https://doi.org/10.30574/wjarr.2021.12.1.0495

\begin{abstract}
Background: The pandemic of COVID-19, a disease caused by novel coronavirus SARS-CoV-2, is associated with significant morbidity and mortality. Recent data showed that hypertension, diabetes mellitus, cardiovascular diseases, and chronic obstructive pulmonary disease were the most prevalent comorbidities in COVID-19 patients. Additionally, data indicate that hypertension, diabetes and cardiovascular diseases are important risk factors for progression and unfavourable outcome in COVID-19 patients. Poorly controlled Type 2 diabetes mellitus was associated with severe progression of disease. Hence, large studies with comprehensive analysis of all risk factors and longer follow-up are necessary.
\end{abstract}

Methods and analysis: A single-centre retrospective cross-sectional study of 300 patients that were SARS-CoV-2 positive from May to October 2020 was done. Data was entered into Microsoft excel data sheet and was analysed using SPSS 22 version software. Chi-square test or Fischer's exact test (for $2 \times 2$ tables only) was used as test of significance for qualitative data. Independent $t$ test was used as test of significance to identify the mean difference between two quantitative variables. ANOVA (Analysis of Variance) was used as test of significance to identify the mean difference between more than two quantitative variables. P value (Probability that the result is true) of $<0.05$ was considered as statistically significant after assuming all the rules of statistical tests.

Data collected was analyzed in a group of patients who were sub divided into 2 groups diabetics and Non diabetics. The parameters studied included laboratory markers: D dimer, CRP, Lactate Dehydrogenase, Ferritin levels. To assess the glycemic control HbA1C levels were assessed. The outcome parameters considered were Oxygen requirement, assessing the requirement of intensive care and duration of stay in the hospital

Conclusion: The patients with higher HbA1c values were found to have higher CRP and D-dimer values and required ICU shift and prolonged hospital stay. Hence, good control of diabetes will reduce the worsening of disease. In a country like India, where most of the population do not have health insurance cover proper control of diabetes, can reduce the burden on family. Hence, large studies with comprehensive analysis of all risk factors and longer follow-up are necessary.

Keywords: Type 2 Diabetes mellitus; COVID-19; Inflammation; Prognosis

\footnotetext{
${ }^{*}$ Corresponding author: Arushi Mohan

Department of Internal Medicine, ASTER CMI Hospital, Hebbal, Bangalore, India.

Copyright $(2021$ Author(s) retain the copyright of this article. This article is published under the terms of the Creative Commons Attribution Liscense 4.0.
} 


\section{Introduction}

A cluster of pneumonia cases of unclear etiology originated in WUHAN city, Hubei province, China, in late December 2019 now known as Coronavirus disease 2019 (COVID- 19) has become a pandemic [1].

The COVID-19 outbreak has led to unprecedented global health crisis, testing health systems preparedness and ability to cope with pandemic response. Its impact is beyond imagination and is sparking a global mourning, booming a feeling of unsafety and uncertainity [2].

The pathogen was identified to be a unique clade from the $\beta$-coronaviruses related to severe acute respiratory syndrome (SARS) and middle east syndrome (MERS), and was officially named severe acute respiratory syndrome coronavirus 2 (SARS-CoV-2). COVID-19 has a higher transmission rate and greater risk of mortality in comparison to influenza [3]. It is transmitted through respiratory droplets and close contacts [4].

Infection with SARS-CoV-2 is characterized by a wide range of clinical presentations from asymptomatic, respiratory manifestations, including flu like symptoms and interstitial pneumonia, which may rapidly progress to acute respiratory distress syndrome (ARDS) requiring admission to intensive care unit (ICU). Other organs like heart, kidney, and liver are also affected and some patients eventually die of multi-organ failure. Ageusia and anosmia are reversible symptoms of COVID-19 [5].

Common manifestations of COVID-19 involve the hematopoietic, hemostatic, and other immune systems and include lymphopenia and hypercoagulability, which correlate with disease severity. Lymphopenia is associated with reduction in total T cells, CD4+ and CD8+ T cell subsets, B cells, and natural killer cells and overproduction of several proinflammatory cytokines, which often may cause a "cytokine storm". Hypercoagulability is evidenced by a characteristic elevation of D-dimer and other fibrin degradation products and by prothrombin time prolongation. Abnormalities in hemostasis may result in widespread thrombosis associated with microvascular injury in the lungs and other affected organs and may even end-up with life-threatening disseminated intravascular coagulation [5].

Among symptomatic individuals hospitalized for COVID-19, older age and presence of comorbidities, including diabetes, obesity, hypertension, chronic obstructive respiratory disease (COPD), cardiovascular disease (CVD), chronic kidney disease (CKD), cancer and immunodeficiency states were shown to be associated with a more severe course and a higher fatality rate.

It is a common chronic metabolic disease, with the influence of bad lifestyle diabetes has become the third largest noncommunicable disease after cardiovascular diseases and tumours [6].

Diabetes mellitus is diagnosed by meeting any one of the following 1) Fasting plasma glucose level $\geq 7.0 \mathrm{mmol} / \mathrm{L} ; 2$ ) Plasma glucose levels $\geq 11.1 \mathrm{mmol} / \mathrm{L} 2$ hours after a 75-gram oral glucose load in glucose tolerance test; 3) Casual plasma glucose level $\geq 11.1 \mathrm{mmol} / \mathrm{L} ; 4)$ Glycated haemoglobin (HbA1c) $\geq 48 \mathrm{mmol} / \mathrm{mol}$ [7].

Diabetes mellitus in general is widely known to cause significant morbidity and mortality as well as health care expenditure [1].

Reviewing literature on the effect of having diabetes in the context of other respiratory viral syndromes such as MERS and SARS show that patients with diabetes have been shown to have higher mortality rate [1].

The presence of diabetes quickly stood out as a major risk factor for increased morbidity and mortality from COVID-19 in China. For example, it was reported that patients with severe COVID-19 and diabetes were significantly more likely to require management in the ICU with mechanical ventilation and ultimately to die from the disease compared to those with severe COVID-19 without diabetes. This observation is supported by a meta-analysis of COVID-19 case series from China. It was found that diabetes was associated with a 2.12-fold increase in mortality, a 2.4-fold increase in severe COVID-19 conditions, and a 4.6-fold increase in acute respiratory distress syndrome (ARDS). There is also a 3.3-fold increase in disease progression from mild condition to more severe stage [7].

In Italy, diabetes was the second most common comorbidity (30\%) after hypertension (67\%) in those who have died from COVID-19 [7]. 


\section{Mechanism Linking Diabetes to Covid-19}

Several mechanisms have been claimed for explaining the exacerbating effect of diabetes on COVID-19. These mechanisms include those related to hyperglycemia and the associated imbalances in pathways involved in virus entry into the cell as well as in immune and inflammatory response. Alternatively, the effect of diabetes may be mediated by diabetes-related comorbidities/complications that have also been associated with poor prognosis [5].

\subsection{Glycemic control}

\subsubsection{Sam riddha Thapa}

Poor glycemic control is a possible risk factor for disease progression in COVID-19. In fact, a retrospective, multicentric survey from Hubei province in China showed that the patients with well controlled blood glucose levels had lower rates of death (1.1\%) and other complications, compared with individuals with poorly controlled blood glucose levels.

The possible diabetogenic effect of SARS-CoV-2 is due to its direct action on key metabolic organs, including the $\beta$ cells of pancreas (Fig: $1 \& 2$ ), they use ACE2 and transmembrane serine protease 2 (TMPRSS2) receptors on $\beta$ cells of pancreas to bind and resulting in new onset hyperglycemia or sudden deterioration of pre-existing diabetes, beyond the well-recognized stress response associated with severe illness. Three reports showed that fasting blood glucose at admission, irrespective of previous diagnosis of diabetes, was an independent predictor of critical illness, death, or poor outcome in patients hospitalized with COVID-19 [5].

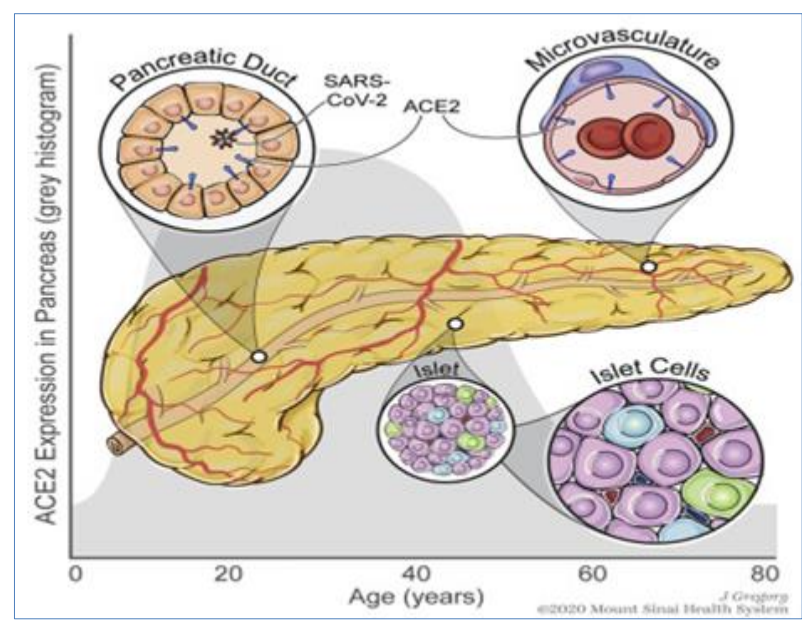

Figure 1 SARS-CoV-2 entry into pancreatic cells.

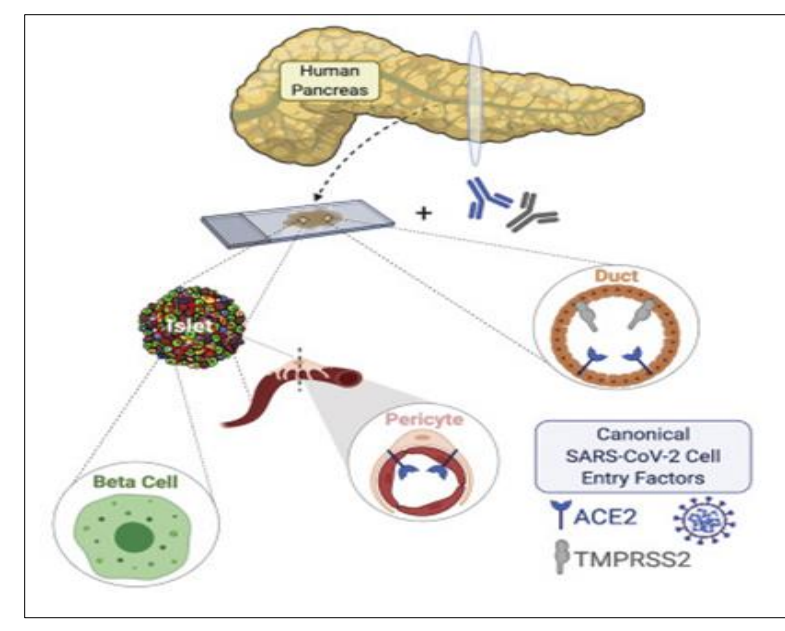

Figure 2 SARS-CoV-2 entry by ACE2 and TMPRSS2 receptors

\subsection{Facilitation of virus entry into the cells}

It has been suggested that diabetes may detrimentally impact the course of the disease through its effects on receptors that mediate virus entry into the cell. Coronavirus receptor proteins include the angiotensin- converting enzyme 2 (ACE2) and DPP4, which are both involved in the regulation of several physiological processes, including glucose metabolism, are modulated by hyperglycemia and pharmacological treatments commonly used in diabetic individuals. Moreover, both receptors exist as a transmembrane and a soluble form, with the latter potentially serving as decoy receptor, which bind and sequesters circulating virus particles. As DPP4 is a receptor for MERS-CoV, but not for SARS-CoV-2, ACE2 has gained most of the attention. A large body of evidence has indicated that treatment with renin-angoiotensin system (RAS) blockers, which increases ACE2 expression, is not associated with either COVID-19 diagnosis or poorer disease outcomes, thus arguing against the hypothesis that the exacerbating effect of diabetes on COVID-19 is mediated through ACE2 upregulation associated with pharmacological RAS blockade. Therefore, discontinuation of these agents is not recommended in COVID-19 patients in order to maintain their anti-hypertensive and cardioprotective effects. (Fig:3) Indeed, it has been postulated that binding of SARS-Cov-2 to ACE2, by reducing the expression and/or activity of this receptor, enhances the vasoconstrictor and pro-inflammatory/pro-oxidant activity of angiotensin II, thus increasing the risk of Acute Lung Injury (ALI). RAS blockade may help mitigate these deleterious effects of angiotensin II.

In addition to RAS activation, diabetes and related comorbidities are also associated with elevated levels of plasmin, a protease that cleaves the S protein of SARS-CoV-2, thus favouring virus binding to ACE2 and entry into cell; moreover, 
fibrin breakdown by plasmin leads to increased levels of D-dimer and other fibrin degradation products, which are characteristic features of severe illness [5].

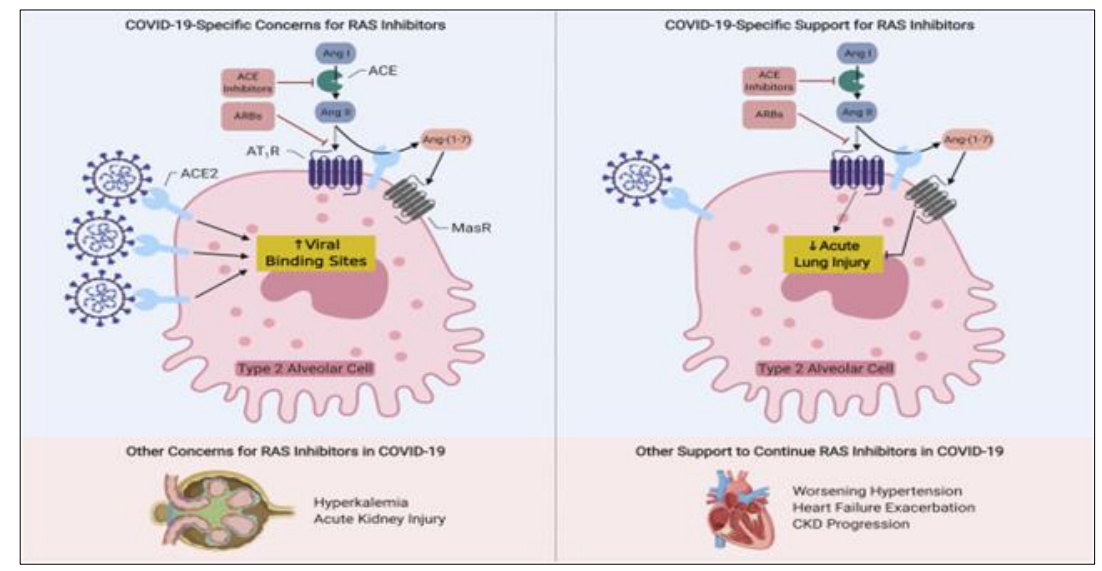

Figure 3 Virus binding to ACE2 receptors, left side showing continuation of RAS inhibitors.

\subsection{Impaired immune and inflammatory response}

Diabetes may also favour the onset and progression of SARS-CoV-2 infection by impairing adaptive immune response to the virus, while enhancing the innate immune system inflammatory reaction. (Fig 4) Diabetes has long been recognized as a risk factor for morbidity and mortality from various types of infections, including those caused by respiratory viruses. In addition, diabetes is also known to be accompanied by a chronic pro-inflammatory and procoagulant state, albeit of low grade, which lead to associated comorbidities/complications. Increased susceptibility to infections has been related to several immune defects, including blunted anti-viral interferon- $\gamma$ response, delayed activation of CD4+ cells with shift toward Th17 responses, and diminished regulatory T cells, which all contribute to hyperinflammation. In patients with COVID-19, immune, inflammatory and coagulation abnormalities were found to be significantly more pronounced in diabetic than in non-diabetic individuals, independently of other comorbidities, and to correlate with glycemic control. As these abnormalities were shown to predict disease severity and adverse outcomes, it was suggested that they may be responsible for exacerbating effect of diabetes [5].

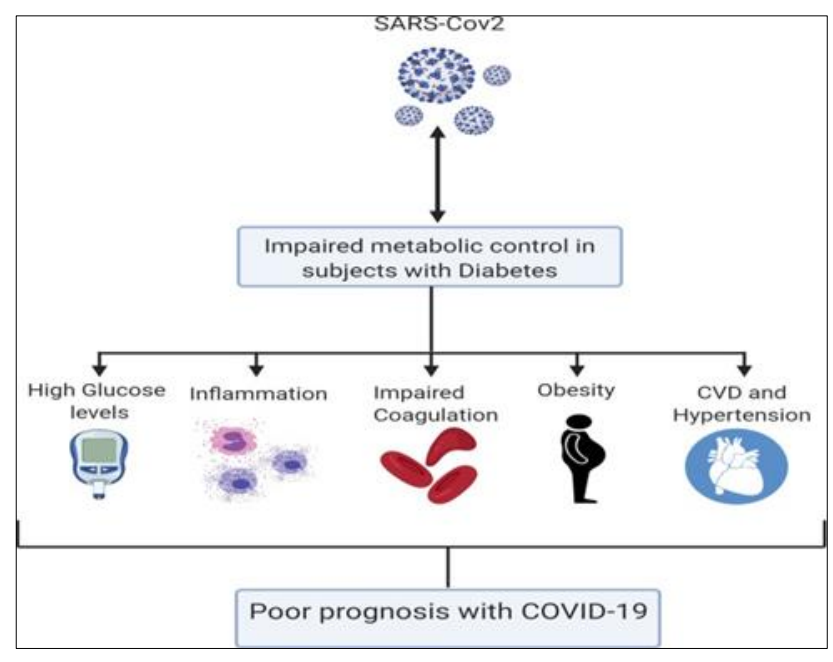

Figure 4 Impact of diabetes and related comorbidities on COVID-19

\subsection{Diabetes related comorbidities}

The negative impact of diabetes on COVID-19 may be related to comorbidities that are frequently associated with it. These comorbidities include obesity, hypertension, which usually cluster with type 2 diabetes (Fig 4) in the context of metabolic syndrome, as well as CVD and CKD, which are common and severe complications of chronic hyperglycemia.

In addition, studies have provided evidence that, just like diabetes, these comorbidities are highly prevalent in COVID19 patients. In particular, hypertension has been shown to be most prevalent comorbidity. In non-Asian patients, 
obesity; in addition to CKD and CVD, including coronary heart disease (CHD), heart failure, and cerebrovascular disease, were also frequently observed in patients with SARS-CoV-2 infection. Among COVID-19 cases reported to the China CDC, prevalence of hypertension and CVD were $12.8 \%$ and $4.2 \%$ respectively [5].

Obesity is another comorbidity associated with poor outcomes after COVID-19 infection, and is associated with poor outcome of COVID-19 infection, and is associated with poor ventilation of the base of the lungs decreasing oxygen saturation of the blood. A recent report from the National Research Center (ICNARC) in the UK demonstrated that out of 196 obese patients with a BMI $>35 \mathrm{~kg} / \mathrm{m}^{2}$ needed mechanical respiration 2 hours after admission. The main factors determining the severity of viral infection in obese obese patients are hormonal environment, the defective response of the innate and adaptive immune system as well as sedentary life style.In summary, obesity and diabetes increases the duration of the disease, the requirement for intensive organ support, and increased risk of mortality [8].

India has a huge number of patients with diabetes; specifically, 18 million patients above 65 years of age who are particularly vulnerable to mortality due to COVID-19. These large number of patients pose a major challenge in prevention and management. Further, during the lockdown, patients with diabetes have increased snacking/carbohydrate intake and decreased physical activity, resulting in weight gain, which may de-stabilize glucose control. A simulation model analysis showed a significant association between the duration of the lockdown and loss of glycemic control as well as associated complications. The predicted increment in $\mathrm{HbA1C}$ from baseline at the end of lockdown was $2.26 \%$. Similarly, the predicted percentage increase in complication rates at the end of lockdown was $2.8 \%$ retinopathy, $9.3 \%$ for microalbuminuria, $14.2 \%$ for proteinuria, $2.9 \%$ for peripheral neuropathy, $10.5 \%$ for lower extremity amputation, $0.9 \%$ for myocardial infarction, and $0.5 \%$ for stroke [7].

Thus, it becomes imperative amidst the current COVID-19 pandemic to investigate the interplay between diabetes and COVID-19 to potentially elucidate treatment strategies and further research opportunities in this specific population. ${ }^{1}$

\subsection{Statistical Analysis}

Data was entered into Microsoft excel data sheet and was analysed using SPSS 22 version software. Categorical data was represented in the form of Frequencies and proportions.

Chi-square test or Fischer's exact test (for $2 \times 2$ tables only) was used as test of significance for qualitative data.

Continuous data was represented as mean and standard deviation. Independent $t$ test was used as test of significance to identify the mean difference between two quantitative variables.

ANOVA was used as test of significance to identify the mean difference between more than two quantitative variables

Graphical representation of data: MS Excel and MS word was used to obtain various types of graphs

$P$ value (Probability that the result is true) of $<0.05$ was considered as statistically significant after assuming all the rules of statistical tests.

Statistical software: MS Excel, SPSS version 22 (IBM SPSS Statistics, Somers NY, USA) was used to analyse data.

\section{Methods and Methodology}

\section{Aim of the study}

- To assess the overall prognosis of hospitalised patients with type 2 diabetes mellitus.

- To see the relationship between HbA1c values and requirement of oxygen and ICU.

- To assess variation of laboratory parameters and severity parameters in the diabetic group.

A single-centre retrospective cross-sectional study of 300 patients that were SARS- CoV-2 positive from May to October 2020 was done.

Data collected was analyzed in a group of patients who were classified as diabetics and Non diabetics. The parameters studied included laboratory markers: D dimer, CRP, Lactate Dehydrogenase, Ferritin levels. To assess the glycemic 
control HbA1C levels were assessed. The outcome parameters considered were Oxygen requirement, assessing the requirement of intensive care and duration of stay in the hospital

\subsection{Inclusion criteria}

Age > 18 years, COVID 19 positive by RT PCR, admitted to the ICU/wards, patients with diabetes, hypertension, chronic kidney disease, coronary artery disease, hypothyroidism and stroke.

Exclusion Criteria: Age $<18$ years, non-COVID, only observed for a day, multiple laboratory parameters missing, no comorbidities, unaware of comorbidities.

\section{Results}

Out of the 300 cases admitted with SARS COV 2,187 were non-diabetic cases and 113 were diabetic cases. The cases admitted with diabetes were more frequent in the elderly age group form 60 years onwards.

Table 1 Distribution of subjects according to age group

\begin{tabular}{|c|c|c|c|c|}
\hline \multirow{2}{*}{} & \multicolumn{2}{|c|}{ Non-Diabetic } & \multicolumn{2}{c|}{ Diabetic } \\
\cline { 2 - 5 } & $\mathbf{N}$ & $\mathbf{\%}$ & $\mathbf{N}$ & $\mathbf{\%}$ \\
\hline 21-30yrs & 30 & $16.0 \%$ & 0 & $.0 \%$ \\
\hline 30-40yrs & 40 & $21.4 \%$ & 8 & $7.1 \%$ \\
\hline 41-50yrs & 43 & $23.0 \%$ & 22 & $19.5 \%$ \\
\hline 51-60yrs & 38 & $20.3 \%$ & 29 & $25.7 \%$ \\
\hline 61-70yrs & 24 & $12.8 \%$ & 38 & $33.6 \%$ \\
\hline 71-80yrs & 9 & $4.8 \%$ & 13 & $11.5 \%$ \\
\hline 81-90yrs & 3 & $1.6 \%$ & 3 & $2.7 \%$ \\
\hline
\end{tabular}

Table 2 Distribution of subjects according to gender

\begin{tabular}{|l|l|l|l|l|}
\hline \multirow{2}{*}{} & \multicolumn{2}{|c|}{ Non-Diabetic } & \multicolumn{2}{l|}{ Diabetic } \\
\cline { 2 - 5 } & $\mathbf{N}$ & $\mathbf{\%}$ & $\mathbf{N}$ & $\mathbf{\%}$ \\
\hline Male & 108 & $57.8 \%$ & 77 & $68.1 \%$ \\
\hline Female & 79 & $42.2 \%$ & 36 & $31.9 \%$ \\
\hline
\end{tabular}

In our study, we had 185 male patients and 115 female patients. In the diabetic group, male were more predominant constituting $68.1 \%$. 
Table 3 Co-morbidities distribution in the patients admitted

\begin{tabular}{|c|c|c|}
\hline & $\mathrm{N}$ & $\%$ \\
\hline HTN & 102 & $33.7 \%$ \\
\hline DM & 113 & $37.3 \%$ \\
\hline CKD & 5 & $1.7 \%$ \\
\hline CAD & 11 & $3.6 \%$ \\
\hline HTN +DM & 59 & $19.5 \%$ \\
\hline HTN +CKD & 5 & $1.7 \%$ \\
\hline HTN +CAD & 8 & $2.6 \%$ \\
\hline HTN +DM +CKD+ HYPOTHYROID & 9 & $3.0 \%$ \\
\hline HTN +DM +CKD & 59 & $19.5 \%$ \\
\hline DM+CAD & 3 & $1.0 \%$ \\
\hline CKD+CAD & 10 & $3.3 \%$ \\
\hline HTN +DM +CAD & 1 & $.3 \%$ \\
\hline HTN+CKD+CAD & 7 & $2.3 \%$ \\
\hline DM +CKD+CAD & 1 & $.3 \%$ \\
\hline HTN+DM+CKD+CAD & 1 & $.3 \%$ \\
\hline Thyroid & 1 & $.3 \%$ \\
\hline TIA & 29 & $9.6 \%$ \\
\hline
\end{tabular}

HTN: hypertension, DM: Type 2 Diabetes mellitus, CKD: Chronic kidney disease, CAD: Coronary artery disease, TIA: Transient Ischemic attack.

In our study, the most prevalent co-morbidity was type 2 diabetes mellitus (37.3\%) followed by hypertension (33.7\%) and CAD (3.6\%).

Table 4 Distribution of symptoms between non-diabetic and diabetic cases

\begin{tabular}{|l|c|c|c|c|}
\hline & \multicolumn{4}{|c|}{ DM } \\
\hline & Non-diabetic & \multicolumn{2}{c|}{ Diabetic } \\
\hline Fever & $\mathrm{N}$ & $\%$ & $\mathrm{~N}$ & $\%$ \\
\hline Breathlessness & 119 & $63.6 \%$ & 67 & $59.3 \%$ \\
\hline Cough & 23 & $12.3 \%$ & 27 & $23.9 \%$ \\
\hline Sore throat & 84 & $44.9 \%$ & 58 & $51.3 \%$ \\
\hline Body ache / tiredness/fatigue & 45 & $24.1 \%$ & 24 & $21.2 \%$ \\
\hline Abdominal pain & 4 & $2.1 \%$ & 2 & $1.8 \%$ \\
\hline Change in stool consistency & 21 & $11.2 \%$ & 14 & $12.4 \%$ \\
\hline Headache & 25 & $13.4 \%$ & 9 & $8.0 \%$ \\
\hline Altered sensorium & 2 & $1.1 \%$ & 3 & $2.7 \%$ \\
\hline loss of taste and smell & 13 & $7.0 \%$ & 6 & $5.3 \%$ \\
\hline
\end{tabular}

In our study, fever and cough were the most common presenting complaints in both diabetics and non-diabetics. Breathlessness as a presenting complaint was observed in $12.3 \%$ of the non -diabetics compared to $23.9 \%$ in the diabetic group.

Bodyache/tiredness/fatigue was seen in $24.1 \%$ in non-diabetic compared to $21.2 \%$ in diabetics. Headache was the presenting complaint in $13.4 \%$ of the non-diabetics compared with $8 \%$ of the diabetics. Sore throat was the presenting symptom in $13.4 \%$ of the non-diabetics compared to $8 \%$ of the diabetics. 
Table 5 Distribution of age groups and shift to the ICU

\begin{tabular}{|l|c|c|c|c|}
\hline \multirow{2}{*}{} & \multicolumn{4}{|c|}{ Shifted to the ICU } \\
\cline { 2 - 5 } & \multicolumn{2}{|c|}{ No } & \multicolumn{2}{c|}{ YES } \\
\cline { 2 - 5 } & $\mathrm{N}$ & $\%$ & $\mathrm{~N}$ & $\%$ \\
\hline $21-30 \mathrm{yrs}$ & 29 & $96.7 \%$ & 1 & $3.3 \%$ \\
\hline 30-40yrs & 44 & $91.7 \%$ & 4 & $8.3 \%$ \\
\hline 41-50yrs & 58 & $89.2 \%$ & 7 & $10.8 \%$ \\
\hline 51-60yrs & 62 & $92.5 \%$ & 5 & $7.5 \%$ \\
\hline 61-70yrs & 56 & $90.3 \%$ & 6 & $9.7 \%$ \\
\hline $71-80 \mathrm{yrs}$ & 20 & $90.9 \%$ & 2 & $9.1 \%$ \\
\hline 81-90yrs & 5 & $83.3 \%$ & 1 & $16.7 \%$ \\
\hline
\end{tabular}

In our study, no statistically significant difference was found between the age groups of patients and their shift to the ICU (p-value 0.906). It indicates that patients of even age below 60 years required shift to ICU if the symptoms and oxygen requirement were worsening.

Table 6 Comparison of ICU shift between diabetic and non-diabetic cases

\begin{tabular}{|l|c|c|}
\hline Shifted to the ICU & Non Diabetic & Diabetic \\
\hline \multirow{2}{*}{ No } & 135 & 32 \\
\cline { 2 - 3 } & $93.1 \%$ & $88.9 \%$ \\
\hline \multirow{2}{*}{ Yes } & 10 & 4 \\
\cline { 2 - 3 } & $6.9 \%$ & $11.1 \%$ \\
\hline \multirow{2}{*}{ Total } & 145 & 36 \\
\cline { 2 - 3 } & $100.0 \%$ & $100.0 \%$ \\
\hline
\end{tabular}

A statistically significant difference was found between non-diabetic and diabetic with respect to their shift to the ICU (p-value 0.034).

Odds ratio $=2.449$, Diabetic subjects are at 2.50 times at risk for getting shifted to the ICU compared to non-diabetic patients. It indicates that diabetic patients are more prone for worsening of symptoms and progression of disease compared to non- diabetic patients.

Table 7 Comparison of HbA1C value and ICU shift

\begin{tabular}{|l|c|c|c|c|}
\hline \multirow{2}{*}{ Shifted to the ICU } & \multicolumn{4}{|c|}{ HbA1c } \\
\cline { 2 - 5 } & $<6.5$ & $6.5-7.5$ & $7.5-8.5$ & $>8.5$ \\
\hline \multirow{2}{*}{ No } & 135 & 32 & 15 & 36 \\
\cline { 2 - 5 } & $93.1 \%$ & $88.9 \%$ & $93.8 \%$ & $85.7 \%$ \\
\hline \multirow{2}{*}{ Yes } & 10 & 4 & 1 & 6 \\
\hline \multirow{3}{*}{ Total } & $6.9 \%$ & $11.1 \%$ & $6.3 \%$ & $14.3 \%$ \\
\hline & 145 & 36 & 16 & 42 \\
\cline { 2 - 5 } & $100.0 \%$ & $100.0 \%$ & $100.0 \%$ & $100.0 \%$ \\
\hline
\end{tabular}


P value 0.4576 , There was no statistically significant difference found between HbA1c and ICU shift. Compared to poorly controlled type 2 diabetes mellitus of $\mathrm{HbA1C}$ more than 8.5, even mild and moderate control of diabetes cases also required ICU shift.

Table 8 Comparison between HbA1C values and oxygen requirement

\begin{tabular}{|l|c|c|c|c|}
\hline \multirow{2}{*}{ Oxygen requirement } & \multicolumn{4}{|c|}{ HbA1c } \\
\cline { 2 - 5 } & $<6.5$ & $6.5-7.5$ & $7.5-8.5$ & $>8.5$ \\
\hline \multirow{2}{*}{ No } & 129 & 33 & 15 & 38 \\
\cline { 2 - 5 } & $89.0 \%$ & $91.7 \%$ & $93.8 \%$ & $90.5 \%$ \\
\hline \multirow{3}{*}{ Yes } & 16 & 3 & 1 & 4 \\
\hline \multirow{3}{*}{ Total } & $11.0 \%$ & $8.3 \%$ & $6.3 \%$ & $9.5 \%$ \\
\cline { 2 - 5 } & 145 & 36 & 16 & 42 \\
\cline { 2 - 5 } & $100.0 \%$ & $100.0 \%$ & $100.0 \%$ & $100.0 \%$ \\
\hline
\end{tabular}

In our study there was no statistically significant difference found between $\mathrm{HbA} 1 \mathrm{C}$ and oxygen requirement, $\mathrm{P}$ value 0.910. In both diabetic and non-diabetic group most of the cases admitted were maintaining saturation in room air. Even in diabetic group cases with mild and moderate control of HbA1C required oxygen similar to poorly controlled HbA1C group of more than $8.5 \%$.

Table 9 Comparison between CRP levels and HbA1c values

\begin{tabular}{|l|c|c|c|c|}
\hline \multirow{2}{*}{ CRP } & \multicolumn{4}{|c|}{ HbA1c } \\
\cline { 2 - 5 } & $<6.5$ & $6.5-7.5$ & $7.5-8.5$ & $>8.5$ \\
\hline \multirow{2}{*}{$<5$} & 66 & 7 & 2 & 6 \\
\cline { 2 - 5 } & $45.5 \%$ & $19.4 \%$ & $12.5 \%$ & $14.3 \%$ \\
\hline \multirow{2}{*}{$5-20$} & 42 & 5 & 3 & 10 \\
\cline { 2 - 5 } & $29.0 \%$ & $13.9 \%$ & $18.8 \%$ & $23.8 \%$ \\
\hline \multirow{2}{*}{$>20$} & 37 & 24 & 11 & 26 \\
\cline { 2 - 5 } & $25.5 \%$ & $66.7 \%$ & $68.8 \%$ & $61.9 \%$ \\
\hline \multirow{2}{*}{ Total } & 145 & 36 & 16 & 42 \\
\cline { 2 - 5 } & $100.0 \%$ & $100.0 \%$ & $100.0 \%$ & $100.0 \%$ \\
\hline
\end{tabular}

In our study there was a significant association between CRP levels and HbA1C values with a $\mathrm{P}$ value $<0.001$. Higher CRP values of 5-20 and $>20$ were found in diabetic patients with poorly controlled diabetes and having HbA1c of $>8.5$, indicating that inflammatory process was more in diabetic patients compared to non-diabetic group. The mean CRP value in diabetic cases was 38.3527.

Table 10 Comparison of D-dimer values and HbA1C values

\begin{tabular}{|l|c|c|c|c|}
\hline \multirow{2}{*}{ D Dimer } & \multicolumn{4}{|c|}{ HbA1c } \\
\cline { 2 - 5 } & $<6.5$ & $6.5-7.5$ & $7.5-8.5$ & $>8.5$ \\
\hline \multirow{2}{*}{$<500$} & 86 & 20 & 9 & 15 \\
\cline { 2 - 5 } & $59.3 \%$ & $55.6 \%$ & $56.3 \%$ & $35.7 \%$ \\
\hline \multirow{2}{*}{$500-1000$} & 39 & 7 & 5 & 14 \\
\cline { 2 - 5 } & $26.9 \%$ & $19.4 \%$ & $31.3 \%$ & $33.3 \%$ \\
\hline \multirow{2}{*}{$>1000$} & 20 & 9 & 2 & 13 \\
\cline { 2 - 5 } & $13.8 \%$ & $25.0 \%$ & $12.5 \%$ & $31.0 \%$ \\
\hline \multirow{2}{*}{ Total } & 145 & 36 & 16 & 42 \\
\cline { 2 - 5 } & $100.0 \%$ & $100.0 \%$ & $100.0 \%$ & $100.0 \%$ \\
\hline
\end{tabular}


$P$ value 0.081 , There was statistically significant difference found between HbA1c and D-dimer indicating that diabetic patients had more tendency of clotting compared to the non-diabetic group. In the diabetic group cases of HbA1C $>8.5$ had higher D-dimer values of more than 1000 indicating higher clotting risk in patients with poorly controlled diabetes. According to our study the mean D-dimer value in diabetic patients was 1974.62.

Table 11 Comparison of HbA1C values and LDH levels

\begin{tabular}{|l|l|l|l|l|}
\hline \multirow{2}{*}{ LDH } & \multicolumn{4}{|c|}{ HbA1c } \\
\cline { 2 - 5 } & $<6.5$ & $6.5-7.5$ & $7.5-8.5$ & $>8.5$ \\
\hline \multirow{2}{*}{$<280$} & 106 & 22 & 10 & 22 \\
\cline { 2 - 5 } & $73.6 \%$ & $61.1 \%$ & $62.5 \%$ & $52.4 \%$ \\
\hline \multirow{2}{*}{$>280$} & 38 & 14 & 6 & 20 \\
\cline { 2 - 5 } & $26.4 \%$ & $38.9 \%$ & $37.5 \%$ & $47.6 \%$ \\
\hline \multirow{2}{*}{ Total } & 145 & 36 & 16 & 42 \\
\cline { 2 - 5 } & $100.0 \%$ & $100.0 \%$ & $100.0 \%$ & $100.0 \%$ \\
\hline
\end{tabular}

In our study, no statistically significant difference was found between HbA1c and LDH. Higher levels of LDH (>280) were found in both diabetic and non-diabetic groups.. The higher levels of LDH of $>280$ was found both in diabetic and non-diabetic cases (P value 0.054). In our study, we found the mean LDH values in diabetic patients as 257.8971.

Table 12 Comparison of HbA1C values and Ferritin levels

\begin{tabular}{|l|l|l|l|l|}
\hline \multirow{2}{*}{ Ferritin } & \multicolumn{4}{|c|}{ HbA1c } \\
\cline { 2 - 5 } & $<6.5$ & $6.5-7.5$ & $7.5-8.5$ & $>8.5$ \\
\hline \multirow{3}{*}{$<250$} & 85 & 21 & 5 & 19 \\
\cline { 2 - 5 } & $58.6 \%$ & $58.3 \%$ & $31.3 \%$ & $45.2 \%$ \\
\hline \multirow{2}{*}{$>250$} & 60 & 15 & 11 & 23 \\
\cline { 2 - 5 } & $41.4 \%$ & $41.7 \%$ & $68.8 \%$ & $54.8 \%$ \\
\hline \multirow{2}{*}{ Total } & 145 & 36 & 16 & 42 \\
\cline { 2 - 5 } & $100.0 \%$ & $100.0 \%$ & $100.0 \%$ & $100.0 \%$ \\
\hline
\end{tabular}

There was no statistically significant difference found between HbA1c and Ferritin with a P value 0.105 . Ferritin levels were high both in diabetic and non-diabetic patients. In diabetic group, patients with HbA1C of more than 7.5 had higher Ferritin levels compared to cases with HbA1C less than 7.5. The mean ferritin levels in our study in diabetic patients was found to be 351.1997 .

Table 13 Comparison of mean and standard deviation between diabetic and non-diabetic patients

\begin{tabular}{|l|c|c|c|c|c|}
\hline \multirow{2}{*}{} & \multicolumn{2}{|c|}{ Non-Diabetic } & \multicolumn{2}{c|}{ Diabetic } & \multirow{2}{*}{ P value } \\
\cline { 2 - 5 } & Mean & SD & Mean & SD & \\
\hline LDH & 282.5804 & 148.0232 & 257.8971 & 92.2346 & 0.124 \\
\hline FERRITIN & 487.3837 & 1202.8644 & 351.1997 & 373.1235 & 0.254 \\
\hline CRP & 46.5321 & 68.5652 & 38.3527 & 54.1669 & 0.282 \\
\hline D-DIMER & 1292.452 & 4858.0577 & 1974.62 & 7766.544 & 0.351 \\
\hline
\end{tabular}


No statistically significant difference was found between Non-Diabetic and Diabetic cases with respect to mean LDH, Ferritin, CRP and D-Dimer.

Table 14 Comparison between diabetic and non-diabetic cases and duration of hospital stay

\begin{tabular}{|l|c|c|c|c|c|}
\hline \multirow{2}{*}{} & \multicolumn{2}{|c|}{ Non-Diabetic } & \multicolumn{2}{c|}{ Diabetic } & \multirow{2}{*}{ P value } \\
\cline { 2 - 5 } & Mean & SD & Mean & SD & \\
\hline No of days in the hospital & 7.83 & 6.408 & 10.86 & 6.964 & $<0.001$ \\
\hline
\end{tabular}

There was a statistically significant difference between the Diabetic and non-Diabetic groups in terms of duration of stay in the hospital. The mean duration of stay of diabetic patients was approximately 11 days compared to around 8 days in non-diabetics.

In our study an analysis done with 300 patients most of the diabetic patients who were admitted to the hospital were elderly with above 60 years of age. Patients admitted were predominantly male patients with a total number of 185 patients out of 300 patients analysed.

In our study, the most prevalent co-morbidity was type 2 diabetes mellitus (37.3\%) followed by hypertension (33.7\%) and CAD (3.6\%). The most common symptoms with which the patient presented with were fever and cough in both diabetic and non-diabetic group.

In our study, we found no statistically difference between age group and shift in ICU; even cases below 60 years of age also required shift to ICU on worsening of symptoms. According to our diabetic subjects were at 2.49 times at risk for getting shifted to the ICU compared to non-diabetic patients, indicating worsening of symptoms and progression of disease in diabetic patients. While comparing the HbA1C value and shift to ICU there was no statistical significance found and even cases with mild and moderate control of diabetes needed shift to ICU.

Majority of cases admitted were maintained saturation at room air in both diabetic and non-diabetic cases. Even in diabetic group cases with mild and moderate control of HbA1C required oxygen similar to poorly controlled HbA1C group of more than $8.5 \%$.

While comparing various inflammatory markers in our study we found that in our study there was significant association between CRP levels and HbA1C values with a P value $<0.001$. Higher CRP values of $5-20$ and $>20$ was found in diabetic patients with poorly controlled diabetes and having HbA1c of $>8.5$, with the mean CRP value of 38.352. On examining the D-dimer values there was statistical significance found. Diabetic patients having HbA1C of more than 6.5 had higher D-dimer values, indicating higher risk of clotting in diabetic patients. While seeing in diabetic population, the patients with $\mathrm{HbA1C}>8.5$ were found to have higher D-dimer values of more than 1000 compared to other groups.

There was no statistical significance found between HbA1C levels and ferritin and LDH levels. But, in the diabetic group the patients with $\mathrm{HbA} 1 \mathrm{C}$ of more than 7.5 were found to have higher ferritin values.

In our study the mean duration of stay for diabetic patients was found to be 10 days while compared to non-diabetic cases.

Hence, in our study we found that diabetic patients were at 2.49 times higher risk to get shifted to ICU and had worsening of symptoms and progression of disease. They were found to have higher CRP values leading to use of higher antibiotics and steroids and remdesivir. Among the diabetic group, the ferritin values were found in patients with $\mathrm{HbA1C}$ of more than 7.5 indicating higher acute inflammation in these patients. In diabetic patients the D-dimer values were higher in cases with $\mathrm{HbA1C}$ of more than 6.5 indicating higher clotting tendencies in these patients. The duration of stay in diabetic patients was also prolonged compared to non-diabetic patients. In a country like India, we have to take in account the financial situation of family as much of the population do not have proper health insurance coverage and cannot afford prolonged stay in hospital.

The GRBS in our setup was monitored $8^{\text {th }}$ hourly and was treated with insulins comprising of both short-acting and long-acting insulins and OHA's. 
Hence proper control of diabetes is very essential to stop the worsening of symptoms and halt the progression of disease.

\section{Discussion}

Individuals with diabetes, especially with type 2 diabetes usually present with metabolic syndrome, i.e. with associated hypertension and/or obesity and higher prevalence of CVD and CKD, as compared with those without diabetes [8].

People with diabetes are not only widely affected by COVID-19 but very often have severe form of the disease. The level of hyperglycemia during the disease can seriously affect the outcome. The survival rate in hospitalized patients with type 2 diabetes with well controlled blood glucose is $99 \%$ [7].

Two nationwide studies by Guan et al, reported a higher prevalence of diabetes among patients with severe versus nonsevere disease (16.2\% vs $5.7 \%$ and $17.7 \%$ vs $6.4 \%$ ). In our study, we found that diabetic patients were at a higher risk to get shifted to ICU.

Zhu et al. reported a higher prevalence of type 2 diabetes in patients with versus without ARDS (25.9\% vs $6.9 \%)$ and in those who died with those who survived (29.8\% vs $12.4 \%$ ). Moreover, ARDS and mortality rate were higher in diabetic versus non-diabetic individuals.

Fadani et al. reported a pooled rate ratio of diabetes among those with more severe versus less severe illness of 2.26 , where Li et al. showed an about two-fold higher proportion of diabetes in ICU/severe cases than in their non-ICU/nonsevere counterparts. Huang et al. showed that diabetes was associated with composite poor outcome and its componenets [5].

Zhou et al reported significantly higher prevalence of diabetes among non-survivors, and they proved that diabetes was associated with mortality from COVID-19.

Wu et al compared COVID-19 patients with and without ARDS and found diabetes was a significant predictor of ARDS.

In a meta- analysis study that included 1558 patients with COVID-19, it was found that diabetes, as well as hypertension and chronic obstructive pulmonary disease (COPD), was associated with exacerbation and admission into intensive care unit.

The majority of inflammatory parameters (C- reactive protein, serum ferritin) and pro-coagulant factors (D-dimer) were also significantly higher among diabetic patients. Similar results were observed in our study. Diabetic complications were more prevalent in patients who died. Interestingly, ketoacidosis and infectious shock, condition that would be assumed to be most responsible for these findings, were not prevalent among COVID-19 patients with lethal outcomes [9].

D-dimer is a fibrin degradation product and is one of the main markers of coagulation activity. The high concentration of serum D-dimer is closely related to a variety of thrombotic diseases, including myocardial infarction, cerebral infarction, pulmonary embolism, and venous thrombosis. In study by Shang et al. they found that the concentration of serum D-dimer of diabetic patients was significantly higher than that of non-diabetic patients, indicating that COVID-19 patients with diabetes are more likely to develop a hypercoagulable pro thrombotic state [3].

In our study, we found a significant association with higher HbA1c values and CRP, D-dimer values indicating inflammatory process occurring more in diabetic patients. But there was no statistical significance noted between HbA1c and LDH values. Higher levels of ferritin and D-dimers were found in cases with HbA1C value of $>7.5$ and $>8.5$ respectively indicating poorer control of diabetes could lead to pro coagulant state leading to complications.

\section{Management of diabetes in COVID-19}

The target blood glucose levels below $10 \mathrm{mmol} / \mathrm{L}$ or $180 \mathrm{mg} / \mathrm{dL}$ has better outcomes. Insulin therapy is the preferred strategy to improve glycemic control in hospitalized patients, and in reducing the risk of ketoacidosis and hypoglycaemia. Insulin schemes either using subcutaneous basal-bolus therapy or intravenous continuous infusion can be used. Insulin doses may be higher than usual due to augmented insulin resistance in these patients. In light of 
mortality benefit from glucocorticoid dexamethasone in those with respiratory failure due to COVID-19, the adoption of appropriate insulin strategies to address steroid-induced hyperglycemia is needed.

Oral anti-diabetes medications can be used in mild COVID-19 cases without severe hyperglycemia as long as they are not contraindicated. There is insufficient evidence to specifically recommend DPP4 inhibitors for the treatment of diabetes in COVID-19. While metformin and SGLT-2 inhibitors may have beneficial effect in heart failure. Furthermore, clinicians should be very cautious about risk of lactic acidosis or ketoacidosis in patients who are severely ill and not eating or hydrating themselves well while on these medications. Dehydration could also impose added risk if patients are on GLP-1 receptor agonists [7]. Among other diabetic medications, the use of liraglutide and pioglitazone have also been found to be related with increased ACE2 regulation in animal studies [10].

Many patients with diabetes also have hypertension, frequently managed with angiotensin receptor blocker (ARBs) or ACE inhibitors. There were early concerns that these medications could interfere with ACE2 to decrease its breakdown, which in turn would facilitate penetration of SARS-CoV-2 into host cells. However, clinical implications is highly questionable that several international organizations have encouraged people to continue to take these medications [3].

In results from Fang et al. patients with diabetes and hypertension who has been treated with ACE inhibitors or ARBs had high numbers of ACE2 receptors in lung, and could therefore be at higher risk of developing severe symptoms [10].

Diabetes facilitates SARS-CoV-2 entry into epithelial cells, as well as development of severe complications resulting in lethal outcome. It is reasonable to hypothesize that pre-existing diabetes-induce target organ damage can be responsible for higher susceptibility of diabetic patients to this virus. Furthermore, diabetic patients with increased inflammatory factors and hyper-coagulability are more likely to progress into a worse prognosis. Therefore, patients with diabetes deserve more intensive attention and should be closely monitored due to the risk of rapid deterioration [5].

\section{Limitation of study}

It is a single centric retrospective study. Obesity and BMI was not taken into account. Data about anti-diabetic therapy at base line are poor. Other inflammatory markers like IL-6 was not done. The study involved only limited number of patients, which can be an important limitation for reaching statistical significance.

\section{Conclusion}

Patients who have type 2 diabetes mellitus and COVID-19 infection had worsening of disease progression. The inflammation and pro-coagulant state are more severe in diabetic patients while compared to non-diabetic patients. They tend to have more severe disease and poorer clinical outcomes. The duration of stay in the hospital is longer and is associated with hypoxia, requiring ICU. Hence, patients with diabetes require special attention and close monitoring. Large studies with comprehensive analysis of all risk factors and longer follow-up are necessary.

\section{Compliance with ethical standards}

\section{Acknowledgments}

The authors would like to thank Sanwal Shah Kalia, Mentor, Acharya Institute of Technology Bengaluru for helping with the data assortment.

Dr Chethan T K, Assistant professor, Department of Community medicine, CIMS, Chamrajnagar for the statistics of the project, Mysore.

\section{Disclosure of conflict of interest}

The authors have no Conflicts of interest to be disclosed

\section{Statement of informed consent}

Not applicable. Institutional ethical clearance was sought. 


\section{References}

[1] Fox T, Ruddiman K, Lo KB, Peterson E, Joy III RD, Salacup G, et al. The relationship between diabetes and clinical outcomes in COVID-19:a single-center retrospective analysis. Acta Diabetol. 2020; 58: 33-38.

[2] Barone MTU, Harnik SB, Luca PV, Lima BLS, Wieselberg RJP, Ngongo B, et al. The impact of COVID-19 on people with diabetes in Brazil. Diabetes Res Clin Pract. 2020; 166: 108304.

[3] Shang J, Wang Q, Zhang H, Wang X, Wan J, Yan Y et al. The relationship between diabetes mellitus and COVID-19 prognosis: A retrospective cohort study in wuhan, China. Am. J. Med. 2020; 000: 1-9.

[4] Hussian S, Baxi H, Jamali M C, Nisar N, Hussian S. Burden of diabetes mellitus and its impact on COVID-19 patients: A meta-analysis of real-world evidence. Diabetes Metab Syndr. 2020; 14: 1595-1602.

[5] Pugliese G, Vitale M, Resi V, Orsi E. is diabetes mellitus a risk factor for coronavirus disease 19 (COVID-19)?. Acta Diabetol. 2020; 57: 1275-85.

[6] Liu Y, Yang Y, Chen Y,Zhou L, Xiong Q, Xie C. The relationship between hyperglycemia and the infection of COVID19 in diabetic patients:A protocol for systemic review and meta-analysis. Medicine 2020; 99: 36(e21806).

[7] Caballero AE, Ceriello A, Misra A, Aschner P, McDonnell ME, Hassanein M et al. COVID-19 in people lieving with diabetes: An international consensus. JDC. 2020; 34.

[8] Muniangi-Muhitu H, Akalestou E, Salem V, Misra S, Oliver N S, Rutter GA. Covid-19 and Diabetes: A complex Bidirectional Relationship. Front. Endocrinol. 2020; 11: 582936.

[9] Tadic M, Cuspidi C, Sala C. COVID-19 and diabetes: Is there enough evidence?. J Clin Hypertens. 2020; 00: 1-6.

[10] Huang I, Lim MA, Pranata R. Diabetes mellitus is associated with increased mortality and severity of disease in COVId-19 pneumonia- A systemic review, meta-analysis, and meta-regression. Diabetes Metab Syndr. 2020; 14 : 395-403. 